\title{
Pathobiology
}

Pathobiology 2017;84:237-242

DOI: $10.1159 / 000471854$
Received: January 24, 2017

Accepted after revision: March 20, 2017

Published online: May 10, 2017

\section{Fatty Acids Induce Stemness in the Stromal Cells of a CT26 Mouse Tumor Model}

\author{
Isao Kawahara ${ }^{a, b}$ Takuya Mori ${ }^{a, b}$ Kei Goto ${ }^{a, c}$ Kiyomu Fujii ${ }^{a}$ Hitoshi Ohmori ${ }^{a}$ \\ Shingo Kishi ${ }^{a}$ Rina Fujiwara-Tani ${ }^{a}$ Hiroki Kuniyasu ${ }^{a}$ \\ ${ }^{a}$ Department of Molecular Pathology, Nara Medical University, Kashihara, ${ }^{b}$ Division of Rehabilitation, Hanna Central \\ Hospital, Ikoma, and 'Division of Rehabilitation, Hoshida Minami Hospital, Katano, Japan
}

\section{Keywords}

Fatty acid $\cdot$ Cancer stroma $\cdot$ Stromal stem cell

\begin{abstract}
The potential effects of 2 types of fatty acids on colorectal cancer (CRC) were assessed using cancer stromal cells. Linoleic acid (LA; C-18, n-6 unsaturated fatty acid) and elaidic acid (EA; C-18, trans acid), both known to affect colon carcinogenesis and cancer progression, were administered by gavage to BALB/c mice, which were inoculated with CT26 syngeneic colon cancer cells in the back. Both EA and LA treatments enhanced tumor growth and metastasis. EA and LA also increased the number of CD133-positive stromal cells in the tumor capsule. Importantly, those cancer cells at the tumor periphery, physically attached to CD133-positive stromal cells, also expressed CD133. These findings suggest that EA and LA might induce stemness in cancer cells through physical association and promote cancer metastasis.
\end{abstract}

C 2017 S. Karger AG, Basel

\section{KARGER}

(C) 2017 S. Karger AG, Basel

E-Mail karger@karger.com

www.karger.com/pat

\section{Introduction}

Cancer stroma is consistent with several types of stromal cells and extracellular matrices, which are associated with cancer cells. Fibroblasts, myofibroblasts, and mesenchymal stem cells (MSC) are a type of cancer stromal cells. Especially, MSC have been known to play an important role in cancer progression. MSC activated by TGF- $\beta$ transdifferentiate to carcinoma-associated fibroblasts, which secrete chemokine-like bioactive substances that enhance metastasis [1]. MSC induce stemness in cancer cells via secretion of CXCL12 or SDF-1 [2-4]. MSC also inhibit the proliferation of T lymphocytes [5] and induce regulatory $\mathrm{T}$ lymphocytes [6-8] to suppress antitumor immunity. Interaction with MSC causes an increase in the metastatic potential of cancer cells. Thus, the number of MSC in cancer stroma is closely associated with the malignant phenotype of cancer cells.

Fatty acids possess a physiological activity that affects cancer cells. Linoleic acid (LA) is an n-6 unsaturated fatty acid with an 18-carbon chain. The procarcinogenic effects of LA on colorectal cancer (CRC) are well known. These include induction of chronic inflammation by the production of prostaglandin E2 [9] or secretion of high-

Hiroki Kuniyasu

Department of Molecular Pathology, Nara Medical University 840 Shijo-cho

Kashihara, Nara 634-8521 (Japan)

E-Mail cooninh@zb4.so-net.ne.jp 
Fig. 1. Effect of elaidic acid (EA) and linoleic acid (LA) on the growth of CT26 subcutaneous (s.c.) tumors. a $\mathrm{BALB} / \mathrm{c}$ mice were treated with either EA or LA (1 mg/ mouse) or vehicle (V; 70\% ethanol) once a week by gavage administration. CT26 cells were inoculated into the subscapular subcutaneous tissue. Body weight (b), tumor weight (c), and number of metastatic nodes in the neck (per mouse) (d) were examined 4 weeks after inoculation. ${ }^{*} \mathrm{p}<0.05$.
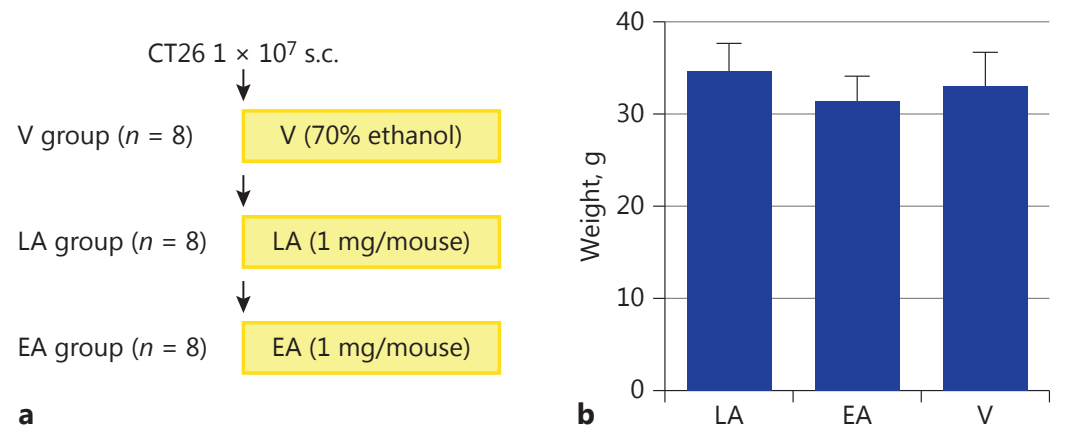

a

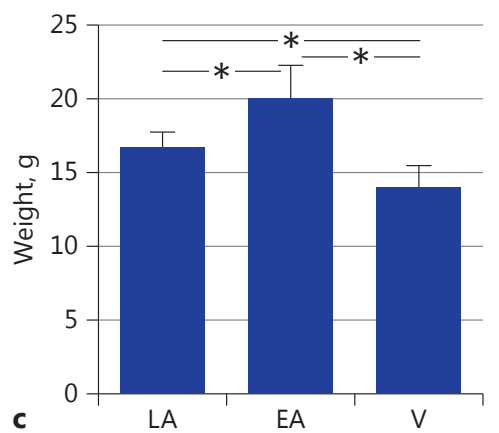

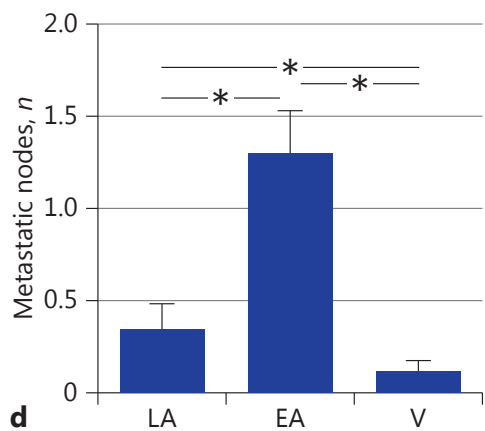

mobility group box-1 [10]. LA is also reported to inhibit peritoneal metastasis and in vitro transformation [11]. Persistent treatment with LA induces dormancy of subcutaneous tumors in mice, which is thought to be associated with a low proliferation of cancer stem cells (CSC) $[10,12]$.

Trans fatty acids (TFA) are mainly generated by industrial hydrogenation and they are used as food constituents, especially in margarine and shortening [13]. TFA consumption is a well-known dietary risk factor for cardiovascular diseases such as atherosclerosis and myocardial infarction $[14,15]$, since it increases the levels of lowdensity lipoprotein-cholesterol and decreases the levels of high-density lipoprotein-cholesterol [16]. In 2015, the FDA issued a final decision that there is no consensus that industrially produced TFA are generally recognized as safe for any use in human food, and that trans fat must be removed from prepared foods within 3 years from this decision, i.e., by June 2018 [17]. TFA are associated with carcinogenesis [18-20]. Moreover, cancer metastasis [21, 22] and drug resistance [23] are promoted by elaidic acid (EA), a major component of dietary TFA [24], through the enhancement of stemness and epithelial-mesenchymal transition $[22,23]$.
In the present study, we aimed to assess the effect of EA on cancer stromal cells in a mouse subcutaneous tumor model.

\section{Materials and Methods}

\section{Cells and Reagents}

The CT26 mouse colon cancer cell line was a kind gift from Prof. I.J. Fidler (MD Anderson Cancer Center, Houston, TX, USA). Cells were cultured in Dulbecco's modified Eagle's medium (Wako Pure Chemical, Osaka, Japan) supplemented with $10 \%$ fetal bovine serum (Sigma Chemical Co., St. Louis, MO, USA).

\section{Animals}

Four-week-old male BALB/c mice were purchased from SLC Japan (Shizuoka, Japan). The animals were maintained in accordance with institutional guidelines approved by the Committee for Animal Experimentation of Nara Medical University, following the current regulations and standards of the Japanese Ministry of Health, Labor, and Welfare (approval No. 9559).

\section{Animal Models}

To establish a subcutaneous tumor model, CT26 cancer cells $\left(1 \times 10^{7}\right)$ were inoculated into the scapular subcutaneous tissue of $\mathrm{BALB} / \mathrm{c}$ mice. Mice were observed for 4 weeks following inoculation. Mice were fed a CE-2 diet (containing 5\% crude fat 


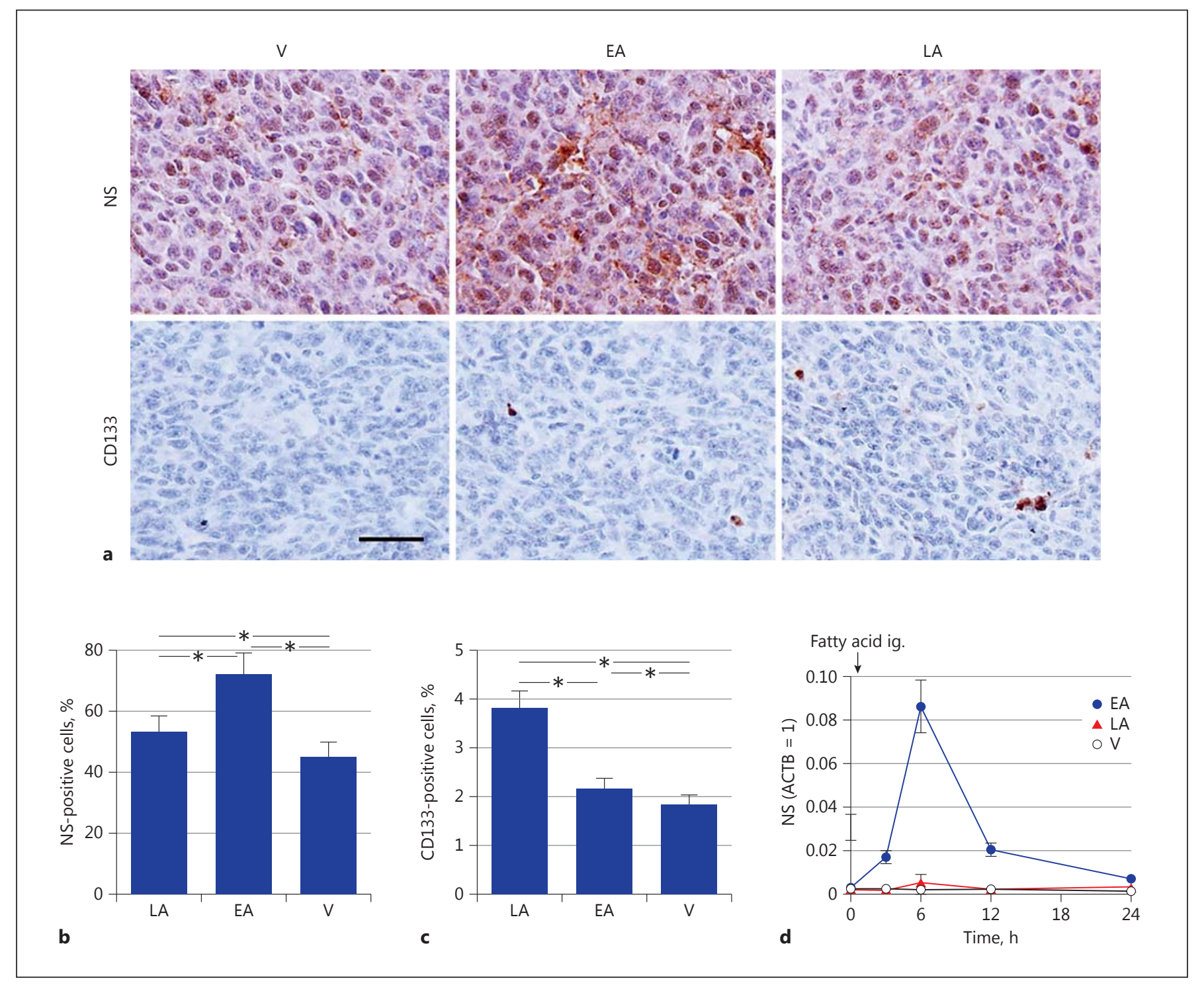

Fig. 2. Effect of elaidic acid (EA) and linoleic acid (LA) on the stemness of CT26 tumors. The expression of nucleostemin (NS) and CD133 in the tumors was examined by immunohistochemistry. Scale bar, $50 \mu \mathrm{m}$. b, c Number of NS-positive and CD133-positive cells, respectively, out of 500 cells counted. $\mathbf{d}$ Relative mRNA levels of NS in mice $(n=3)$. Error bars represent SD. ${ }^{*} \mathrm{p}<0.05$. V, vehicle; ig., intragastric gavage administration.

derived mainly from soybean oil; CLEA Japan, Inc., Tokyo, Japan). EA (1 mg/mouse; Sigma) or LA (1 mg/mouse; Wako) diluted with vehicle (70\% ethanol) was administered by gavage every 7 days.

\section{Immunohistochemistry}

Consecutive $4-\mu \mathrm{m}$ sections were immunohistochemically stained using the immunoperoxidase technique, as described previously [25]. Antibodies against mouse CD133 (Proteintech, Rosemont, IL, USA), and nucleostemin (NS; Santa-Cruz Biotechnology, Inc., Dallas, TX, USA) were used at $0.5 \mu \mathrm{g} / \mathrm{mL}$. Color development was achieved using 3-3'-diaminobenzidine (DAKO,
Carpinteria, CA, USA). Specimens were counterstained with Meyer's hematoxylin stain (Sigma) to visualize the nuclei. After immunostaining, the number of positively stained nuclei was counted under a microscope on all slides.

For immunofluorescent staining, primary antibodies were labeled with fluorescent dye by using the specific labeling kit for each dye (ThermoFisher Scientific, Yokohama, Japan). Antibodies for CD133 (Proteintech), CD73 (Abnova Corp., Taipei City, Taiwan), and AE3 (Santa-Cruz) were labeled with Alexa Fluor 488, Alexa Fluor 568, and Alexa Fluor 350, respectively. DAPI (Santa-Cruz) was used for nuclear staining. The images were captured using an all-in-one microscope (Keyence, Osaka, Japan). 
Fig. 3. Effect of elaidic acid (EA) and linoleic acid (LA) on the interaction between stromal and cancer cells. a The expression of CD133 at the tumor periphery was examined by immunohistochemistry. Scale bar, $50 \mu \mathrm{m}$. EV, enlarged view. Scale bar, $20 \mu \mathrm{m}$. FI, immunohistochemistry with CD133 (stem cell marker; Alexa Fluor 488, green), CD73 (bone marrow mesenchymal cell marker; Alexa Fluor 568, red), AE3 (epithelial cell marker; Alexa Fluor 350, blue), and DAPI (nuclear marker). b Number of CD133-positive stromal cells out of 200 cells counted. c Number of CD133-positive cells at the tumor periphery out of 200 cells counted. ${ }^{*} \mathrm{p}<0.05$. HPF, high-power field.

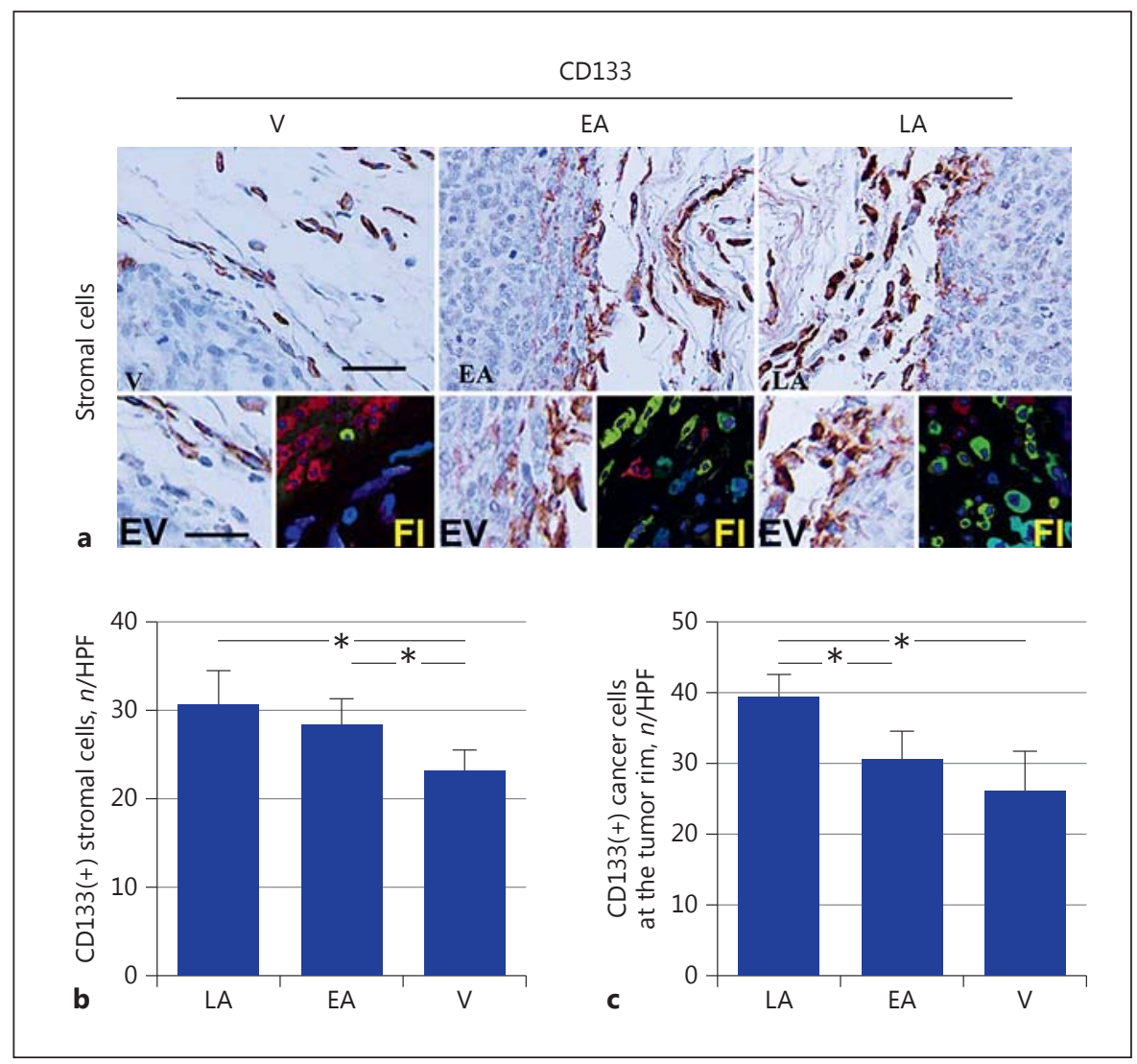

Reverse Transcription-Polymerase Chain Reaction

Total RNA (1 $\mu \mathrm{g})$ was synthesized using a PAX gene Blood RNA Kit (Qiagen, Valencia, CA, USA). Blood was collected by cardiocentesis after anesthesia from 3 mice in each group. Polymerase chain reaction (PCR) products were confirmed on $2 \%$ agarose gel and visualized with ethidium bromide. The following primer sets (Sigma Genosys, Ishikari, Japan) were used for amplification: mouse NS (Gln3, accession No. AY181025.1): forward 5'-CAG GAT GCT GAC GAT CAA GA-3', reverse 5' ${ }^{\prime}$-TTG ATT GCT CAG GTG ACA GC- $3^{\prime}$, and mouse $\beta$-actin (ActB, accession No. NM_007393.4): forward 5'-AGC CAT GTA CGT AGC CAT CC-3', reverse 5' ${ }^{\prime}$-CTC TCA GCT GTG GTG GTG AA-3'. PCR conditions were set in accordance with the manufacturer's instructions.

The quantitative reverse transcription (RT)-PCR was performed using QuantiTect Primer Assays (Qiagen) and the readydesigned primers following the manufacturer's instructions. The assay is based on the detection of RT-PCR products by SYBR green.

\section{Statistical Analysis}

Statistical significance was calculated using unpaired Student $t$ tests with InStat software (GraphPad, Los Angeles, CA, USA). Statistical significance was defined as a 2 -sided $p$ value $<0.05$.

\section{Results}

\section{Effect of EA and LA on the Growth of CT26}

\section{Subcutaneous Tumors}

CT26 cells were inoculated into the subscapular subcutaneous tissue of syngeneic BALB/c mice, which were also administered either EA or LA (Fig. 1a). No statistical difference was observed in the weight of mice among the LA, EA, and control groups (Fig. 1b). Tumor weight and the number of nodal metastases were highest in the EA group, followed by the LA group (Fig. 1c, d).

\section{Effect of EA and LA on the Stemness of CT26 Tumors}

The effects of LA and EA on the stemness of CT26 cells were assessed by examining NS and CD133 expression (Fig. 2a-c). The number of NS-positive cells was highest in the EA group, followed by the LA group. In contrast, the number of CD133-positive cells was highest in the LA group, followed by the EA group.

NS mRNA was detected by RT-PCR in the blood of mice treated with EA as well as in that of mice treated with LA. EA treatment increased NS mRNA in the blood $12 \mathrm{~h}$ 
after gavage; however, administration of LA or vehicle did not alter NS mRNA levels in the blood.

\section{Effect of EA and LA on the Interaction between}

\section{Stromal and Cancer Cells}

Immunohistochemistry of the tumors showed that CD133-positive stromal cells surrounded the tumor periphery (Fig. 3a). The number of CD133-positive stromal cells was highest in the LA group, followed by the EA group (Fig. 3b). Interestingly, both the LA and EA groups showed CD133-positive stromal cells attached to cancer cells expressing CD133 at the tumor periphery (Fig. 3a, enlarged view). Most of the stromal cells expressed CD73, a bone marrow mesenchymal cell marker. Multifluorescent immunohistochemistry showed that CD133+/CD73+ stromal cells increased with an increase in CD133+/AE3+ cancer cells via treatment with LA or EA. In contrast, a physical association between cancer and stromal cells was not found in the vehicle group. The number of CD133-positive cancer cells at the tumor periphery was higher in the LA and EA groups than in the vehicle group (Fig. 3c).

\section{Discussion}

In the present study, we examined the effects of LA and EA, which possess reactivity to CSC, on tumor stromal cells. The most important finding is that both LA and EA enhanced the stemness of stromal cells as well as the physical attachment between cancer cells and stromal cells.

We showed that treatment with LA and EA promoted tumor growth and nodal metastasis. The prometastatic effect of LA and EA could be explained by the enhancement of stemness in cancer cells. Moreover, our data showed that LA and EA possessed reactivity to cancer stromal cells. LA and EA treatment increased the number of CD133-positive stromal cells in the tumors. These cells are compatible with MSC, which might have originated from bone marrow-derived MSC or induced topical MSC.

CT26 mouse colon cancer cells express NS and CD133; however, each positivity is different. NS is expressed in many cells, whereas CD133 is expressed in a few cells. NS expression is closely associated with CD133 in breast cancer cells [26]. The difference in positivity between NS and CD133 might be reflected by their recognition of the range of stemness. Our previous data suggest that NS recognized CSC, progenitor cells, and proliferating cells, whereas CD133 recognized CSC [21]. The increase in NSpositive cancer cells might be associated with proliferative stem cells, which are suspected to possess a high met-

Fatty Acids Enhance Cancer Stromal Cell Stemness astatic potential. Moreover, LA upregulates CD133, whereas growth inhibition is provided from the activation of PPAR $\gamma$ [27]. LA metabolites act as ligands of PPAR $\gamma$. These differences between LA and EA affect their capacity to promote metastability.

Cell-to-cell attachment between stromal cells and cancer cells at the tumor periphery was observed in mice treated with LA and EA. This interaction is known to promote the metastatic ability of cancer cells by causing an increase in cancer stemness [2-4], acquisition of the epithelial-mesenchymal transition phenotype via secretion of CXCR4, SDF-1, or TGF- $\beta$ from stromal stem cells [28], induction of regulatory $\mathrm{T}$ cells by stromal stem cells [24], fusion of cancer cells with stromal stem cells [29], and multicellular sphere formation of cancer cells and stromal cells [30].

The findings of the current study are consistent with those of our previous studies, which showed that metastatic potential is located at the tumor periphery $[31,32]$. We previously demonstrated that cancer cells at the tumor periphery express metastasis-associated genes, such as EGFR and MMP-2/-9, and repress E-cadherin [31,32], which might be triggered by CSC induction at the tumor periphery.

In this study, we showed that fatty acids affect mesenchymal cells in cancer stroma; however, our data suggest that fatty acids exert direct bioactive effects on cancer stromal cells, which might be considered a CSC niche, in which interactions between cancer cells and stromal cells increase stemness in both types of cells. The precise mechanism underlying the enhancement of stemness through the cancer-stroma physical interaction is a subject of further study.

\section{Acknowledgements}

The authors thank Ms. Tomomi Masutani for her expert assistance with the preparation of this paper. This work was supported by MEXT KAKENHI grant No. 16675788.

\section{Disclosure Statement}

We declare that there is not any financial support or relationships which may pose a conflict of interest in the contents of the submitted manuscript. All authors have approved the comments.
Pathobiology 2017;84:237-242

DOI: $10.1159 / 000471854$ 


\section{References}

1 Barcellos-de-Souza P, Comito G, Pons-Segura C, Taddei ML, Gori V, Becherucci V, Bambi F, Margheri F, Laurenzana A, Del Rosso M, Chiarugi P: Mesenchymal stem cells are recruited and activated into carcinoma-associated fibroblasts by prostate cancer microenvironment-derived TGF- $\beta_{1}$. Stem Cells 2016; 34:2536-2547.

2 Corcoran KE, Trzaska KA, Fernandes H, Bryan $\mathrm{M}$, Taborga $\mathrm{M}$, Srinivas V, Packman $\mathrm{K}, \mathrm{Pa}$ tel PS, Rameshwar P: Mesenchymal stem cells in early entry of breast cancer into bone marrow. PLoS One 2008; 3:e2563.

3 Duda DG, Kozin SV, Kirkpatrick ND, Xu L, Fukumura D, Jain RK: CXCL12 (SDF1a)CXCR4/CXCR7 pathway inhibition: an emerging sensitizer for anticancer therapies? Clin Cancer Res 2011;17:2074-2080.

4 Mimeault M, Batra SK: Frequent gene products and molecular pathways altered in prostate cancer- and metastasis-initiating cells and their progenies and novel promising multitargeted therapies. Mol Med 2011;17:949964.

5 Fontaine MJ, Shih H, Schafer R, Pittenger MF: Unraveling the mesenchymal stromal cells' paracrine immunomodulatory effects. Transfus Med Rev 2016;30:37-43.

6 Duffy MM, Ritter T, Ceredig R, Griffin MD: Mesenchymal stem cell effects on T-cell effector pathways. Stem Cell Res Ther 2011;2:34.

7 Luz-Crawford P, Kurte M, Bravo-Alegria J, Contreras R, Nova-Lamperti E, Tejedor G, Noel D, Jorgensen C, Figueroa F, Djouad F, Carrion F: Mesenchymal stem cells generate a CD4+CD25+Foxp3+ regulatory $\mathrm{T}$ cell population during the differentiation process of Th1 and Th17 cells. Stem Cell Res Ther 2013; 4:65.

8 Patel SA, Dave MA, Bliss SA, Giec-Ujda AB, Bryan M, Pliner LF, Rameshwar P: Treg/Th17 polarization by distinct subsets of breast cancer cells is dictated by the interaction with mesenchymal stem cells. J Cancer Stem Cell Res 2014;2014:e1003.

9 Singh J, Hamid R, Reddy BS: Dietary fat and colon cancer: modulation of cyclooxygenase- 2 by types and amount of dietary fat during the postinitiation stage of colon carcinogenesis. Cancer Res 1997;57:3465-3470.

10 Ohmori H, Sasahira T, Fujii K, Luo Y, Shimomoto T, Kuniyasu H: Linoleic acid-induced growth suppression induces quiescent cancer cell nests in nude mice. Pathobiology 2008;75: 226-232.

11 Kuniyasu H: Linoleic acid; in Schwab M (ed): Encyclopedia of Cancer, ed 3. New York, Springer, 2008.
12 Luo Y, Chihara Y, Fujimoto K, Sasahira T, Kuwada M, Fujiwara R, Fujii K, Ohmori H, Kuniyasu $\mathrm{H}$ : High mobility group box 1 released from necrotic cells enhances regrowth and metastasis of cancer cells that have survived chemotherapy. Eur J Cancer 2013;49: 741-751.

13 Food and Drug Administration: Tentative determination regarding partially hydrogenated oils: request for comments and for scientific data and information. 2013. http://www. fda.gov/NewsEvents/Newsroom/PressAnnouncements/ucm373939.htm.

14 Remig V, Franklin B, Margolis S, Kostas G, Nece T, Street JC: Trans fats in America: a review of their use, consumption, health implications, and regulation. J Am Diet Assoc 2010;110:585-592.

15 Mozaffarian D, Aro A, Willett WC: Health effects of trans-fatty acids: experimental and observational evidence. Eur J Clin Nutr 2009; 63(suppl 2):S5-S21.

16 Dashti N, Feng Q, Franklin FA: Long-term effects of cis and trans monounsaturated (18:1) and saturated (16:0) fatty acids on the synthesis and secretion of apolipoprotein A-I- and apolipoprotein B-containing lipoproteins in HepG2 cells. J Lipid Res 2000;41:1980-1990.

17 Food and Drug Administration: Final determination regarding partially hydrogenated oils. 2015. https://www.federalregister.gov/ articles/2015/06/17/2015-14883/final-determination-regarding-partially-hydrogenatedoils.

18 Vinikoor LC, Schroeder JC, Millikan RC, Satia JA, Martin CF, Ibrahim J, Galanko JA, Sandler RS: Consumption of trans-fatty acid and its association with colorectal adenomas. Am J Epidemiol 2008;168:289-297.

19 Slattery ML, Benson J, Ma KN, Schaffer D, Potter JD: Trans-fatty acids and colon cancer. Nutr Cancer 2001;39:170-175.

20 Pickens CA, Lane-Elliot A, Comstock SS, Fenton JI: Altered saturated and monounsaturated plasma phospholipid fatty acid profiles in adult males with colon adenomas. Cancer Epidemiol Biomarkers Prev 2016;25:498506.

21 Fujii K, Luo Y, Fujiwara-Tani R, Kishi S, He S, Yang S, Sasaki T, Ohmori H, Kuniyasu H: Pro-metastatic intracellular signaling of elaidic acid, a trans fatty acid. Int J Oncol 2016;50:85-92.
22 Ohmori H, Fujii K, Kadochi Y, Mori S, Nishiguchi Y, Fujiwara R, Kishi S, Sasaki T, Kuniyasu $\mathrm{H}$ : Elaidic acid, a trans-fatty acid, enhances the metastasis of colorectal cancer cells. Pathobiology 2016, E-pub ahead of print.

23 Tanabe E, Kitayoshi M, Fujii K, Ohmori H, Luo Y, Fujiwara R, Kadochi Y, Mori S, Kuniyasu $\mathrm{H}$ : Fatty acids inhibit anti-cancer effect of 5-fluorouracil in mouse cancer cell lines. Oncol Lett 2016, E-pub ahead of print.

24 Precht D, Molkentin J: Trans fatty acids: Implications for health, analytical methods, incidence in edible fats and intake (a review). Nahrung 1995;39:343-374.

25 Kuniyasu H, Yano S, Sasaki T, Sasahira T, Sone S, Ohmori H: Colon cancer cell-derived high mobility group 1/amphoterin induces growth inhibition and apoptosis in macrophages. Am J Pathol 2005;166:751-760.

26 Lin T, Meng L, Li Y, Tsai RY: Tumor-initiating function of nucleostemin-enriched mammary tumor cells. Cancer Res 2010;70:94449452.

27 Sasaki T, Fujii K, Yoshida K, Shimura H, Sasahira T, Ohmori H, Kuniyasu H: Peritoneal metastasis inhibition by linoleic acid with activation of PPAR $\gamma$ in human gastrointestinal cancer cells. Virchows Arch 2006;448:422427.

28 Mele V, Muraro MG, Calabrese D, Pfaff D, Amatruda N, Amicarella F, Kvinlaug B, Bocelli-Tyndall C, Martin I, Resink TJ, Heberer M, Oertli D, Terracciano L, Spagnoli GC, Iezzi G: Mesenchymal stromal cells induce epithelial-to-mesenchymal transition in human colorectal cancer cells through the expression of surface-bound TGF-beta. Int J Cancer 2014;134:2583-2594.

29 Melzer C, Yang Y, Hass R: Interaction of MSC with tumor cells. Cell Commun Signal 2016; 14:20.

30 Duda DG, Duyverman AM, Kohno M, Snuderl M, Steller EJ, Fukumura D, Jain RK: Malignant cells facilitate lung metastasis by bringing their own soil. Proc Natl Acad Sci USA 2010;107:21677-21682.

31 Kuniyasu H, Ellis LM, Evans DB, Abbruzzese JL, Fenoglio CJ, Bucana CD, Cleary KR, Tahara E, Fidler IJ: Relative expression of E-cadherin and type IV collagenase genes predicts disease outcome in patients with resectable pancreatic carcinoma. Clin Cancer Res 1999; 5:25-33.

32 Kuniyasu H, Yasui W, Pettaway CA, Yano S, Oue N, Tahara E, Fidler IJ: Interferon-alpha prevents selection of doxorubicin-resistant undifferentiated-androgen-insensitive metastatic human prostate cancer cells. Prostate 2001;49:19-29. 